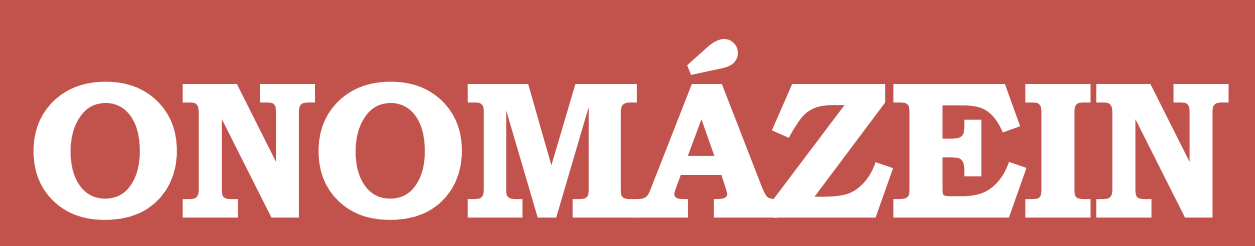

Revista de lingüística, filología y traducción
PONTIFICIA UNIVERSIDAD

\title{
La construcción resultativa del inglés en contraste
}

The English resultative construction in contrast

\author{
Luis París \\ INCIHUSA-CONICET / UNCUYO \\ Argentina
}

ONOMÁZEIN 46 (diciembre de 2019): 259-286

DOI: 10.7764/onomazein.46.13

ISSN: 0718-5758

\section{(C) (1) $\odot$}

Luis París: INCIHUSA-CONICET / UNCuyo, Argentina. | E-mail: paris@mendoza-conicet.gob.ar 


\section{Resumen}

Este estudio tiene dos objetivos enmarcados en el establecimiento de contrastes entre lenguas mediante una gramática de interfaces. El primero es describir la estructura característica de la construcción resultativa del inglés (KR), analizar una muestra representativa de sus subtipos y explicitar las condiciones sintáctico-semánticas que la hacen posible. KR es una Macrosituación que fusiona dos Situaciones - una Actividad y un Evento - en la secuencia de un 'curso causal de Situaciones' tal que el Evento es el Resultado de la Actividad. Alcanzar esta caracterización requiere subsumir Manera bajo Actividad, Resultado bajo Cambio y CAUSA bajo 'curso causal de Situaciones'. El segundo objetivo es exponer los factores que habilitan la presencia de KR en inglés y que solo permiten una escueta presencia en español. KR es la Macrosituación complementaria de la Situación prototípica del inglés, Situación que se opone a la del español ya que presenta una Actividad como verbo principal mientras la otra proyecta un Evento. KR no es complementaria en el sistema español. Además, KR instancia el estilo combinatorio ‘abierto' característico del inglés —y opuesto al 'cerrado' del español_tal que permite un conjunto abierto de relaciones semánticas entre dos predicados, mientras el español tiende a reducirlas idealmente a una.

Palabras clave: construcción resultativa; contraste inglés-español; interfaz sintaxis-semántica; Situación; Macrosituación.

\section{Abstract}

This paper has two intertwined objectives that are consistent with the overall goal of using an interface grammar to describe different contrasts among languages. The first one is to describe the internal structure of the English resultative construction or $\mathrm{KR}$, to characterize a representative sample of the KR subtypes and to render explicit the syntax-semantics interface conditions that make it possible. $K R$ is a Macroevent that fuses two Events-an Activity and an Event proper-in the temporal sequence of a 'causal course of Events' so that this Event is the Result of the Activity. In order to reach this characterization, it is necessary to subsume Manner under Activity, Result under Change and CAUSE under 'causal course of Events'. The second objective is to expose the features that license the presence of KR in English while permitting a scarce presence of it in Spanish. KR is the Macroevent that complements the prototypical English Event, which opposes to Spanish in so far as it projects an Activity as main verb while the Spanish Event projects an Event proper. Thus, KR is not a 
complementary structure for the Spanish Event. Furthermore, KR reflects the typical 'open' combinatory English style, which is quite different from the Spanish 'close' one. This means that English allows for an open set of semantic relations between two words under the same structure while Spanish prefers to reduce them to (likely) one.

Keywords: resultative construction; Spanish-English contrast; syntax-semantics interface; Event; Macroevent. 


\section{Introducción}

La sofisticación de las representaciones cognitivas que un sujeto genera de su entorno resulta de un proceso creativo que tiene como raíz la lengua que adquirió, usa y transforma. Esta lengua contiene un conjunto de piezas léxicas que constituyen categorías de cosas, propiedades y situaciones que en español se agrupan, respectivamente, como nombres, adjetivos y verbos. El hablante segmenta su experiencia con estas categorías con un grado de fineza que es directamente proporcional a la riqueza de su léxico. La lengua provee, además, un conjunto de transformadores - morfología derivativa - y precisadores - morfología flexiva- de categorías y es crucial que también ofrezca esquemas combinatorios de esas categorías y esquemas que unifican esquemas que resultan finalmente en una oración. La oración es el símbolo que en definitiva le permite al sujeto captar y expresar una experiencia que es inherentemente temporal y que, por lo tanto, necesita ser segmentada en Situaciones ${ }^{1}$. Cada Situación conlleva un conjunto de participantes - típicamente individuos - que interactúan y adoptan una función distintiva y específica. Esta interacción se extiende a lo largo del tiempo tal que cada Situación es una entidad temporal en el estricto sentido de tener sus partes distribuidas a lo largo de diferentes lapsos de un mismo intervalo.

Resulta esencial considerar que la Situación es un constructo lingüístico que solo viene a ser respaldado por la realidad. La Situaciónı es confirmada por la [[situación $\left.{ }_{1}\right]$ ] en el mundo extralingüístico ${ }^{2}$. Este dato es especialmente determinante para este estudio ya que la misma [[situación]] en el mundo puede respaldar descripciones lingüísticas alternativas en distintas lenguas o, más sorprendente aún, en una misma lengua. Los filósofos captan este hecho con el rótulo de ‘subdeterminación’: una misma realidad extralingüística puede hacer verdaderas descripciones divergentes. Por ejemplo, las oraciones (1) y (2) describen la misma [[situación]] en la que un panadero saca de un camión una bandeja con pan y la lleva a la panadería.

(1) El panadero entró al negocio con una bandeja de pan.

(2) El panadero entró al negocio cargando una bandeja de pan.

$1 \quad$ Si bien la noción de Evento aparece como más apropiada en el sentido de tener arraigadas en el uso coloquial las nociones de dinamismo y temporalidad, voy a adoptar el término Situación como equivalente para disipar la ambigüedad que resulta del uso de Evento en la literatura semántica como un subtipo de Situación al lado de, por ejemplo, Estados y Actividades.

2 Utilizo la convención de doble corchete para referirme a una entidad del mundo, por ejemplo, un

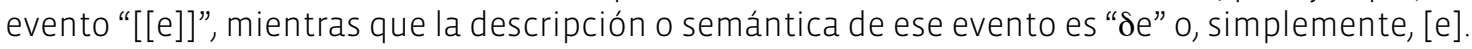
Esta descripción puede referir tanto a la semántica esquemática de un verbo (descripción de un tipo o clase de evento) o a la descripción saturada introducida por una oración que describe un evento individual. Esta distinción es aquí poco relevante y, por lo tanto, la omito. Los intervalos o trazas temporales de cada evento son representados como "t $\mathrm{t}_{\mathrm{e}}$. 
Podemos analizar estos datos en múltiples direcciones pero la relevante aquí es que estas dos oraciones no son sinónimas aun cuando representen la misma [[situación]] en el mundo. Es un caso de sinonimia 'solapada'. En particular, la relación entre el panadero y la bandeja en (1) es estática e inespecífica y solo reinterpretada como temporal en relación a la Situación introducida por el verbo principal "entrar". Por el contrario, (2) construye esa relación como una Situación: Ia semántica léxica de "cargar" conlleva una relación entre participantes que duró un lapso de tiempo durante el cual un participante ejerció activamente una fuerza direccionada al otro participante. La realidad es la misma pero las descripciones son diferentes aunque ambas verdaderas; (1) introduce una Situación simple, en cambio (2) contiene una Macrosituación: una Situación compleja en la que tiene que resolverse la relación no léxica entre dos Situaciones ([entrar] y [cargar]).

La construcción resultativa del inglés — de aquí en más KR — representa la constitución de un tipo de Macrosituación. Una Macrosituación consiste en el enriquecimiento de una Situación mediante un constituyente que introduce una (sub)Situación en el marco de una sintaxis reducida. Esto es, las Situaciones simples se corresponden con oraciones simples; en cambio, una Macrosituación involucra dos cláusulas estrechamente dependientes tal que la expresión formal de la cláusula subsumida está dramáticamente menguada. KR encierra un complejo entramado sintáctico-semántico que aporta una pieza particularmente original al repertorio de formas con las que las lenguas registran y estructuran la experiencia. La oración (3) representa una instancia típica de KR.

(3) John danced Liz tired.

John bailó Liz cansada

"John cansó a Liz de tanto bailar/bailando"

El verbo matriz es originalmente intransitivo (John danced last night "John bailó anoche"). Denota una Situación dinámica y abierta (una Actividad) con un único argumento expresado como sujeto de la oración. Esta semántica léxica carece de un argumento afectado; sin embargo, en la instancia de KR en (3), Liz es el Paciente de un Cambio de Estado indicado por tired y, más aún, es realizado como objeto directo de un verbo intransitivo. Se debe subrayar que se trata de un objeto directo que, al contrario de los postulados de (quizás) todas las teorías gramaticales, no es habilitado por la semántica léxica del verbo. Dance no contiene - como tampoco "bailar" - en su significado un participante afectado en el sentido en el que lo está Liz en (3). El uso de la noción de ‘construcción' para caracterizar a KR se justifica tanto en esta habilitación como en la correspondiente introducción de nociones semánticas estructurales como Resultado y Cambio. Se trata de una particular asociación de forma con un significado no enteramente composicional que, además, es (parcialmente) productiva.

KR revela con particular claridad el subtipo característico de las Macrosituaciones del inglés, arquitectura manifiesta también, entre otras, en las construcciones 'way' y 'away' en (4) y (5) tanto como la versión de KR con un verbo perifrástico ejemplificada en (6). 
(4) Trump wormed his way to the Presidency.

"Trump llegó a la presidencia moviéndose como un gusano"

(5) Let's twist the night away.

Dejemos torcer la noche afuera

"Reventemos la noche"

(6) Strega Nona talked my warts off.

Bruja Abuela habló mis verrugas afuera

"La Abuela Bruja me sacó las verrugas hablando"

Dada una [[situación]] que pueda ser captada por una Actividad (Situación dinámica y abierta) o un Evento (Situación cerrada y télica), el estilo inglés expresa la Actividad como verbo principal. Aquello que el Actor y sujeto de la oración hace es descripto con particular riqueza y especificidad y es erigido como centro de la oración. Esta Actividad permite un potencial cierre mediante la determinación de su efecto, información que es introducida por un elemento léxico subordinado al verbo principal como lo mostró el análisis del ejemplo (3) pero que también es válido para los casos (4), (5) y (6). En (4) el verbo describe una particular forma de desplazamiento (como un gusano) a la que la construcción añade un Trayecto (his way) y su Meta (to the Presidency). En (5) el verbo principal describe lo que hace el Actor a la vez que también especifica cómo el Paciente es afectado mientras que el elemento léxico away codifica el Resultado de esa Actividad en términos de un Cambio de Estado. El verbo en (6) es intransitivo y denota una Actividad que es especificada por el contenido introducido por una partícula (off) y que afecta a un objeto directo sin habilitación léxica: la Actividad [talk] denotada por talk causa el Cambio en un Paciente - my warts.

La Macrosituación como constructo gramatical se sustenta en la subespecificación: en cierto sentido, las formas lingüísticas no codifican toda la información que la realidad extralingüística denotada acarrea. De aquí que toda forma pueda enriquecerse semánticamente en distintas direcciones mediante, por ejemplo, la introducción de (sub)Situaciones. Las características de la Macrosituación en una lengua dependen, entonces, del estilo arquitectónico de su Situación prototípica. El inglés es una lengua de marco satelital (Talmy, 2000). Resulta evidente a partir de las traducciones de (3) a (6) que la estructura arquetípica del inglés es enteramente extraña al español. Mi posición aquí es que se trata de un caso de traducción 'solapada' en el sentido de que dos oraciones pueden referir a la misma [[situación]] pero definitivamente no tienen el mismo significado, sino que son solo parcialmente equivalentes. El español es una lengua de marco verbal, es decir, la Situación típica codifica al Resultado en el verbo principal y no en un elemento adjunto.

Un corolario del contraste tal cual ha sido explicitado arriba es que el inglés se focaliza en describir la Actividad del Actor - y, por lo tanto, del sujeto- mientras el español prefiere especificar la afectación del Paciente y, por lo tanto, del objeto directo (París, 2016). El enri- 
quecimiento natural de la Situación inglesa consiste en la Macrosituación KR que incluye un Resultado, típicamente en términos de un Cambio de Estado o Cambio Locativo, que especifique lo que le sucedió al Paciente, y esto es precisamente lo que presenta KR. París (2015) razona de manera funcionalista para explicar la menguada presencia de KR en español: sería redundante contar con una forma que enriqueciera la Situación española con una Macrosituación que añada el Resultado. Por el contrario, la Macrosituación típica del español —con una presencia muy limitada en el inglés - es la codificada por la construcción adverbial de gerundio ejemplificada en las traducciones de (3) y (4) en las que al Evento de Cambio de Estado introducido por el verbo principal se le añade una Actividad del Actor mediante una frase de gerundio (París, 2018).

Esta investigación tiene dos objetivos. El primero es comprender KR, esto es, describir su semántica y sintaxis, la interface entre ambas y el rol distintivo que ocupa en el mosaico de recursos expresivos del inglés. Con este propósito dedico la siguiente sección a presentar los subtipos más representativos de KR. La exposición de la semántica de KR me lleva a revisar nociones fundacionales como la de CAUSA, Manera y Telicidad. Mi análisis concluye que la noción de causalidad involucrada en KR es la de un mismo 'curso causal de Situaciones', una noción más general que la de CAUSA típica de la semántica léxica. A su vez, Manera (entre Situaciones) es derivada de Actividad y, finalmente, propongo que la noción de Cambio, más específica que la de Telicidad, es la relevante para caracterizar KR.

Mi segundo objetivo es identificar al factor que habilita la presencia de KR en inglés y que hace lánguida su presencia en español. La idea básica es que KR es semántica y formalmente consistente con el sistema gramatical inglés y, por el contrario, es inconsistente con el del español. En general, presupongo un universal tipológico que prohíbe la presencia de todo el espectro de subtipos de KR en lenguajes de marco verbal como el español. A su vez, asumo una presencia poco productiva de construcciones con predicados verbales con función adverbial tal como la construcción adverbial de gerundio del español en lenguas de marco satelital. Por un lado, la investigación que presento aquí fundamenta la explicación compensatoria de naturaleza semanticista en París (2015) sobre la ocurrencia de KR en inglés y su ausencia en español y, por otro, explicita las propiedades estructurales de KR —específicamente, de la interfaz sintaxis-semántica - para resaltar que exhibe rasgos formales enteramente ausentes en el español: la presencia de un objeto directo no habilitado por el verbo principal.

En relación al análisis semántico este trabajo profundiza y justifica la propuesta en París (2015) al enraizar la tipología de Talmy en una disyunción revisada de la lexicalización de Manera y Resultado propuesta en Rappaport y Levin (2010). En tal sentido, se evalúa críticamente la noción de Manera para proponer su subsunción en la categoría de Actividad y se subsume, a su vez, Resultado bajo Cambio. Es así que reinterpreto la disyunción Manera-Resultado como Actividad-Cambio. Esto me obliga a especificar los dominios donde la disyunción está activa y aquellos en los que no tiene injerencia y, más importante aún, a proponer un criterio que permi- 
ta delimitar esos dos dominios: la disyunción Actividad-Cambio se funda en una condición cognitiva, la ‘segregación'. Esta condición desalienta la combinación Actividad-Cambio por razones de economía léxica y referencialidad. La propuesta es que al asociar una Actividad y Cambio bajo un mismo verbo, el espectro de uso de ese verbo queda exponencialmente limitado. Se requerirían muchos más ítems léxicos verbales para registrar el mismo espacio semántico potencialmente cubierto por un solo verbo de Actividad sin Cambio especificado. La confluencia sistemática de Actividad (Manera) y Cambio (Resultado) se vuelve enteramente antieconómica.

En relación al contraste formal, mi propuesta es que KR instancia una estructura inexistente en español: dos predicados léxicos 'plenos' en un único dominio sintáctico clausal. Es una estructura que representa lo más cercano que una lengua puede llegar a las construcciones seriales sin contar con estas; se trata de combinar sintácticamente dos verbos con el valor de uno respecto de su comportamiento formal. La evidencia más clara de la presencia de esta estructura es el objeto directo en (3) habilitado por el adjetivo tired y por la noción de Cambio en una misma cadena causal ${ }^{3}$.

En la siguiente sección presento una muestra representativa del abanico de variaciones que subsume KR. En la tercera sección discuto la representación adecuada de KR y analizo los factores que posibilitan su existencia y determinan sus propiedades fundamentales. En la cuarta presento mi propuesta sobre el rol de KR en el contraste inglés-español. En la quinta discuto propuestas alternativas y, finalmente, resumo la propuesta en la conclusión.

\section{EL abanico de Macrosituaciones KR}

KR no debería ser interpretada estrictamente como una construcción, sino como una familia de (sub)construcciones (Goldberg y Jackendoff, 2004; Rappaport y Levin, 2001) o, como la he venido presentando aquí, una familia de Macrosituaciones. Este constructo teórico nos permite captar la identidad de un patrón de relaciones semántico-sintácticas sin asumir que solo una forma específica o una semántica particular están involucradas. Un subtipo frecuente de KR es el representado en (3), que repito a continuación, y en el que la expresión resultativa es la frase adjetiva FA tired ("cansada").

3 La noción de predicado 'pleno' remite a una forma verbal o adjetiva cuyo significado tiene valor referencial, es decir, denota una [[situación]] o una [[propiedad]] tal que pueden ser usados como núcleos de una cláusula independiente (verbo) o de una frase adjetiva. En el caso de KR, tanto el verbo como el adjetivo hacen la misma contribución semántica que haría ese verbo o adjetivo en una oración que no fuese una instancia de KR. En cambio, los denominados verbos livianos o bien no aparecen solos en cláusulas independientes o, si lo hacen, no tienen el mismo significado que cuando son usados como livianos. El español sí tiene la estructura $V_{1}-V_{2}$ inherente a $K R$, pero solo si $V_{1}$ es un verbo liviano como en la construcción causativa 'hacer-infinitivo'. En síntesis, no admite $V_{1}-V_{2}$ con dos verbos plenos. 
(3) John danced Liz tired.

John bailó Liz cansada

"John dejó a Liz cansada de tanto bailar"

La descripción de la Situación matriz $\left[\mathrm{e}_{\mathrm{M}}\right]$ es introducida por el verbo intransitivo dance. Se trata de una Actividad, es decir, una Situación abierta, relativamente homogénea y dinámica. La descripción del Evento Resultativo $\left[\mathrm{e}_{\mathrm{R}}\right.$ ] es aportada por una FA que introduce un Estado, es decir, una Situación estática, homogénea y abierta que deviene un Cambio de Estado (y, por ende, un Evento) al insertarse como último eslabón del curso causal de Situaciones al interior de la Macrosituación KR. Es decir, el significado de KR no puede derivarse simplemente de la composición de los significados léxicos. Los ítems léxicos solo aportan dos descripciones de Situaciones, una Actividad y un Estado; sin embargo, la interpretación de (3) demanda además la satisfacción de las siguientes restricciones:

(7) a. Una relación temporal de precedencia $\left[\left[e_{M}\right]\right]$ y $\left[\left[e_{R}\right]\right]$ es tal que $t_{M}<t_{R}$.

b. Una relación causal entre $\left[\left[\mathrm{e}_{M}\right]\right]$ y $\left[\left[\mathrm{e}_{R}\right]\right]$ tal que $\left[\left[\mathrm{e}_{\mathrm{M}}\right]\right]$ CAUSA $\left[\left[\mathrm{e}_{\mathrm{R}}\right]\right]$.

c. FA aporta un Estado [[s]] que en KR deviene un Cambio de Estado [[e $\left.e_{R}\right]$ ] causado por [[ $\left.e_{M}\right]$.

d. Ese Cambio de Estado es predicado de un participante codificado en la cláusula matriz (Liz).

El intervalo $\left[\left[\mathrm{t}_{M}\right]\right]$ asociado a $\left[\left[\mathrm{e}_{M}\right]\right]$ precede al intervalo $\left[\left[\mathrm{t}_{R}\right]\right]$ asociado a $\left[\left[\mathrm{e}_{R}\right]\right]$. El Cambio de Estado implica que Liz no estaba cansada —o, al menos, no estaba tan cansada — antes de bailar y resultó cansada luego: Liz adquirió una propiedad luego de bailar; es un cambio discreto que va de no A a A. Por último, este Cambio afectó necesariamente a Liz y no se predica de John.

(8) [[John-bailar-con-Liz ${ }_{M}$ ] CAUSA [BECOME [Liz-estar-cansada $\left.{ }_{R}\right] \&_{1}<t_{2}$

El sentido en el que la Macrosituación KR constituye una 'construcción' es que una de sus partes se sigue de principios generales de composición e interfaz sintáctico-semántica mientras otra es construccional. No solo los elementos léxicos conllevan semántica, sino que la estructura combinatoria en sí está asociada también a un significado.

Desde el punto de vista formal, el verbo matriz "dance” es, como su equivalente español, intransitivo y puede transitivizarse si añadimos un Tema como "tango".

(9) Rob danced a tango.

"Rob bailó un tango"

Liz no podría ser Tema (incremental) de dance porque no satisface las restricciones semánticas que le impone el verbo al participante en ese rol: ser una pieza musical. Un participante humano como el denotado por Liz es típicamente Acompañante en ese contexto; de hecho, en español usaríamos "con" para introducirlo. Sin embargo, en (9) funciona como objeto directo. 
Se trata, por un lado, de una característica saliente y, por otro, de una restricción de la construcción. KR introduce un argumento ausente en la semántica léxica del verbo; Liz es afectada de un modo no entrañado por la semántica léxica del verbo, su estado de cansancio no es añadido a la ocurrencia de la Situación. Además, es objeto directo en el dominio sintáctico local del verbo principal. Las teorías gramaticales asumen que todo objeto directo es habilitado por la semántica léxica del verbo tal que aparece en su estructura argumental con un rol semántico asignado. KR demanda, entonces, un tratamiento descriptivo excepcional. Este fenómeno se correlaciona con la restricción DOR (por Direct Object Restriction), formulada originalmente en Levin y Rappaport (1995) y refrendada últimamente en Levin y Rappaport (2019). KR exigiría a la cláusula matriz un objeto directo que, a su vez, exprese al argumento del que se predica el Cambio de Estado. No se trata del objeto directo superficial, sino del 'profundo' o, como diríamos en teorías no transformacionales, del argumento Afectado (Undergoer). De aquí que con verbos intransitivos inacusativos, el Resultado puede ser predicado del sujeto porque no se trata de un Actor sino de un argumento Afectado.

(10) The snow frozed solid.

La nieve congeló sólida

"La nieve se congeló hasta ponerse dura"

No es irrelevante que DOR sea capaz de ser la motivación que deriva la presencia de falsos reflexivos como en (11).

(11) Sam coughed himself hoarse.

Sam tosió a sí mismo ronco

"Sam quedó ronco de tanto toser"

El verbo "cough" es intransitivo. El reflexivo "himself” no satisface ninguna función semántica en el Evento [[cough]]. Sin embargo, es obligatorio, como lo muestra (12). Su presencia solo se explica como un recurso para satisfacer DOR.

(12)*Sam coughed hoarse.

En contra del imperio de DOR como regla inflexible, existen ciertos pares mínimos que exhiben tanto la presencia como la ausencia del reflexivo.

(13)a. Sam wiggled free.

Sam serpenteó libre

"Sam se liberó contorsionando"

b. Sam wiggled himself free.

Sam serpenteó a sí mismo libre

"Sam se liberó contorsionando" 
Se sugiere un contraste semántico entre los dos ejemplos. Mientras (13a) denotaría un único Evento, (13b) expresa dos Eventos potencialmente separados en el tiempo (Levin y Rappaport, 2019).

Si bien en KR la relación causal es directa —es decir, se asume que no existe un evento entre $\left[\left[e_{M}\right]\right]$ y $\left[\left[e_{R}\right]\right]$ 一, la relación temporal no necesita serlo. En (14) puede haber habido un lapso considerable de tiempo entre el envenenamiento y la muerte, pero de manera crucial se asume que ningún evento relevante para la muerte sucedió entre ellos. Se exige contigüidad de Eventos pero no contigüidad temporal.

(14) Belle poisoned Kyle dead.

Belle envenenó Kyle muerto

"Belle mató a Kyle envenenándolo"

La reformulación de la restricción en términos de argumento Afectado (Undergoer) no podría tampoco salvar DOR de contraejemplos como (15) o, más devastador aún, (16).

(15) The three kings followed the star out of Bethlem.

Los tres reyes siguieron la estrella afuera de Belén

"Los tres reyes salieron de Belén siguiendo la estrella"

(16) Peter ran into the classroom.

Peter corrió en-a el aula

"Peter entró al aula corriendo"

En ambos casos el Resultado es un Cambio Locativo predicado del Actor - no Afectado- del verbo principal y que deviene Afectado solo por la predicación resultativa. En síntesis, DOR resulta una condición que, si bien transgredible, tiende a ser satisfecha en KR.

Los casos (15) y (16) representan un subtipo particular de KR ya que el componente resultativo no es registrado por una frase adjetiva FA, sino por una expresión satelital o una frase preposicional. En ambos casos, este componente está asociado a un Trayecto (Path) que registra también su punto final Meta pero que, además, construye esta Meta como un Cambio Locativo que involucra el traspaso de un límite (out o into). No se trata solo de alcanzar el final del Trayecto, sino de un Cambio de Lugar. El ejemplo (17) muestra que la expresión resultativa asociada a un Trayecto puede expresar también un Cambio de Estado.

(17) They rely on pictures of baby seals being clubbed to death.

Ellos basan en fotos de bebé focas siendo apaleadas hasta muerte

"Ellos se basan en fotos de ballenatos que son apaleados hasta la muerte"

Washio (1997) denomina Resultativa Fuerte a la subclase de KR que subsume a todos los ejemplos analizados hasta aquí y contiene incluso casos como el de (18), donde de la Actividad matriz tiende a lograr — pero no entraña - el Resultado descrito por la FA. 
(18) Cori wiped the table clean.

Cori repasó la mesa limpia

"Cori limpió la mesa repasándola”

La Actividad matriz no entraña necesariamente al Resultado. De hecho, es posible negar ese Resultado sin contradicción, como en (19).

(19) Cori wiped the table but it wasn't clean after all.

"Cori repasó la mesa pero no estaba limpia después de todo"

Por el contrario, en las resultativas débiles la Actividad entraña el Resultado que la FA solo se limita a especificar. El cambio de estado [[devenir-rojo]] en (20) especifica un Resultado que a partir de "pintar" no podía ser otro que un color. Ese Resultado está altamente restringido y, por lo tanto, es una KR débil.

(20) Edward painted the door red.

Edward pintó la puerta roja

"Edward pintó la puerta roja"

El verbo matriz describe una Actividad que, por la combinación con una frase nominal delimitada - "the door" - deviene una Realización que incorpora la noción de Cambio de Estado. KR solo específica aquí ese Cambio, no lo introduce.

\section{La estructura de KR}

Una Macrosituación materializa lo que se ha denominado Event Augmentation ('Desarrollo de un Evento'). La precondición para la existencia de Macrosituaciones es la vaguedad intrínseca de toda forma lingüística: la forma - el verbo principal de la oración - no codifica toda la información de la Situación que describe, por lo cual siempre puede ser enriquecida. Una Macrosituación conlleva la presencia de al menos dos predicados plenos que aluden a Situaciones que tienen un vínculo semántico en relación a un mismo intervalo de tiempo y que comparten, además, un mismo participante. La relación semántica en KR es la de 'curso causal de Situaciones' - similar a la CAUSA léxica- y los predicados plenos son instanciados por un verbo o adjetivo o una frase preposicional. La restricción sintáctica es que las formas que expresan las Situaciones sean morfosintácticamente independientes y se combinen entre sí al interior de un dominio sintáctico local, es decir, léxico. En el caso de KR, la simetría o asimetría de los predicados es relativa. Por un lado, la expresión resultativa es sintácticamente más simple y dependiente de operadores expresados en el verbo principal; por otro lado, se trata a veces de un elemento necesario cuya ausencia tornaría la oración en agramatical (*John danced Mary “* John bailó a Mary”). En otras palabras, a pesar de no ser un complemento, la forma subordinada se incrusta profunda y estrechamente en la cláusula principal, lo que 
significa que tiene un elevado grado de dependencia sintáctica en términos de operadores temporales, negación, modalidad, modo e interpretación semántica.

Propongo que a KR subyace una estructura sintáctica con un único núcleo o ‘juntura nuclear' (nuclear juncture [Van Valin, 2005]).

\section{FIGURA 1}

La sintaxis de KR

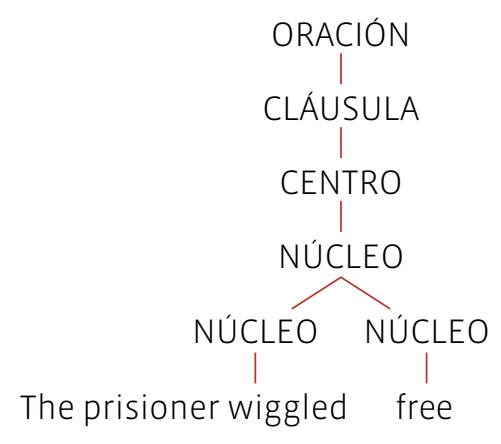

Sin espacio para intentar una justificación basada en un detallado análisis sintáctico, la razón fundamental de este análisis fue ya introducida: Ia relación semántica cuasi léxica entre los predicados plenos y, más importante aún, la introducción de un objeto directo no léxicamente habilitado en el dominio sintáctico del verbo principal y que registra, además, un participante compartido por ambos predicados. No existe otro modo de dar cuenta de esta relación si no es admitiendo que los dos predicados se combinan al interior de un mismo dominio sintáctico en el que comparten sus argumentos.

La semántica de KR fue resumida en (7). Involucra relaciones semánticas típicamente codificadas al interior de una semántica léxica verbal que, por definición, conlleva una única aunque potencialmente compleja-Situación. Se trata de una relación de precedencia entre dos eventos tal que uno causa al otro. Además, el [EventoR] es un Cambio de Estado/Locativo causalmente provocado por [Situación $n_{M}$, típicamente una [Actividad].

Esta Macrosituación es constituida por una relación de adición (Krifka, 1998). Se trata de un tipo de adición más estricta que la general y en la que $\left[\left[\mathrm{e}_{R}\right]\right]$ se añade a $\left[\left[\mathrm{e}_{\mathrm{M}}\right]\right]$ respetando la precedencia temporal y la restricción sobre el 'curso causal de Situaciones'.

4 Esta precedencia no necesita ser total, puede haber un solapamiento de trazas temporales - los intervalos asociados a cada evento - tal que solo la parte inicial de $\mathrm{t}_{\mathrm{M}} \mathrm{O} I \mathrm{NI}\left(\mathrm{t}_{\mathrm{M}}\right)$ preceda estrictamente a $t_{R}$.

(i) $\left.\left(I N I\left(t_{M}\right)\right)<t_{R}\right)$ 
(21) $\left[\left[e_{M z}\right]\right] \oplus\left[\left[e_{R}\right]\right]$

Existen matices de (7) que necesitan ser revisados. No es obvio que la relación causal en KR sea idéntica a la que se les asigna a los verbos causativos (como "matar" o "romper"). La causalidad en la semántica de un verbo entraña dos sub-Situaciones - una causante, la otra efecto - tal que, si seguimos la definición de Lewis (1973), el efecto no tendría lugar sin la presencia previa del causante. Si pensamos en “matar”, expresado en (22), los entrañamientos relevantes se listan en (23).

(22) La fiebre amarilla mató a tres turistas.

(23) a. La fiebre amarilla es Actor en una Situación [[ $\left.\left.e_{1}\right]\right]$.

b. Tres turistas estaban vivos y resultaron muertos, un Cambio de Estado $\left.\left[\mathrm{e}_{2}\right]\right]$.

c. $\left[\left[\mathrm{e}_{1}\right]\right]$ precede temporalmente a $\left[\left[\mathrm{e}_{2}\right]\right]$.

d. $\left[\left[e_{1}\right]\right]$ causó $\left[\left[e_{2}\right]\right]$.

Aun cuando $\left[\left[e_{1}\right]\right]$ no sea descrita ni especificada, es posible distinguir claramente dos subSituaciones, $\left[\left[e_{1}\right]\right]$ y $\left[\left[e_{2}\right]\right]$, y esta distinción es tanto temporal como basada en que el Cambio de Estado del Paciente - el efecto - es cualitativamente distinto a la sub-Situación [ $\mathrm{e}_{1}$ ] que contiene al Actor. En los ejemplos de KR denominados débiles (Washio, 1997), esta distinción no es necesaria. Por ejemplo, en (24) el Cambio de Estado está presente en la oración simple $y$, por ende, no puede ser atribuido a KR en (25).

(24) Peter painted the door.

"Peter pintó la puerta"

(25) Peter painted the door red.

"Peter pintó la puerta roja"

La oración simple no es causativa en el sentido léxico. En (22) un evento causativo no especificado precede al efecto; en cambio, en (24) el evento ejecutado por el Actor es claramente especificado, tanto que el verbo permite un uso intransitivo de pura Actividad (en español, "Pedro pintó durante una hora"). La semántica de (24) es captada mediante una Actividad que, si el Afectado es delimitado, culmina en un Cambio de Estado final, una Realización. Siendo esta la semántica de "pintar" - como efectivamente se asume en la literatura relevante-, proponer que la versión KR de paint es causativa aparece como inmotivado. La noción apropiada aquí es la de eventos en ‘un mismo curso' o Path (Krifka, 1998; París, 2006b). La diferencia central es la siguiente:

(26) a. En un 'curso causal de Situaciones', las Situaciones están en una misma cadena causal pero, al contrario de la causalidad léxica, no se les exige que estén en una secuencia estricta tal que una preceda totalmente a la otra. 
b. Por el contrario, las Situaciones en un mismo curso pueden solaparse. Es lo que sucede en todas las Realizaciones: el Cambio de Estado es parte de un proceso de afectación precedente, es el último punto de ese proceso.

c. La presencia de Temas Incrementales convierte al punto 'b' en evidente: en (24) y (25) la puerta es afectada incrementalmente a lo largo de un proceso que termina cuando esa afectación es total. Esta afectación gradual es correlativa con la Actividad del Actor.

En síntesis, la causalidad depende del tipo de KR, puede ser tanto la del curso de eventos como la causalidad léxica típica. Finalmente, necesitamos subrayar que la noción Telicidad no es suficiente para caracterizar la semántica de KR, se requiere de Cambio de Estado. El ejemplo (27) es télico pero no es una instancia de KR.

(27) John walked to the Seven Eleven to buy some bread.

John caminó a el almacén para comprar algo de pan

"John caminó al almacén a comprar algo de pan"

En este caso una Actividad (walk) recorre un Trayecto delimitado. Un ejemplo de KR con verbo de movimiento involucra, además, un Cambio Locativo, es decir, el cruce de un límite (Aske, 1989: 6). En (28) no solo hay un Trayecto delimitado, sino que se establece el traspaso de un límite: el Tema va más allá del límite que encierra un interior y lo distingue del exterior.

(28) John walked out of the house.

John caminó afuera de la casa

"John salió de la casa caminando"

Este cruce de límites distingue a un Cambio Locativo de la Telicidad en general y es la noción que requiere KR; el Cambio Locativo es télico pero exige además el traspaso de un Límite. Puesto que la Telicidad es captada en la literatura por el primitivo DEVENIR, propongo reemplazarlo en (7) por RESULTADO, al que interpreto como una versión más fuerte que exige Cambio Locativo / de Estado 5 .

Una propiedad esencial para comprender KR remite a las limitaciones de su productividad. Boas (2000: 301) analiza un corpus de diez millones de palabras y encuentra, entre otros hechos, que solo catorce adjetivos ocurren más de tres veces en el contexto de KR. Solo cinco aparecen más de diez veces (flat, apart, awake, open, shut). El estudio de Boas muestra, entonces, que KR permite un conjunto restringido de adjetivos. Lo mismo podría concluirse de las expresiones satelitales en general, de lo cual inferimos la productividad muy restringida de KR.

5 En realidad, el término BECOME para captar Telicidad es inapropiado a la luz de lo que se sabe hoy, porque fue definido originalmente como 'Cambio' y, en realidad, se trata de una categoría más general que involucra solo un Límite (natural). 
Las propiedades sobresalientes de la KR prototípica pueden ser resumidas con las siguientes. KR describe una Macrosituación en la que se especifica una sub-Situación inicial que causa una sub-Situación Resultado en una secuencia de intervalos contiguos. El verbo principal expresa la sub-Situación inicial y esta es típicamente una Actividad. El Resultado es o bien una propiedad télica (escala cerrada) expresada en una FA o bien el Lugar que delimita, a su vez, un Trayecto expresado por una FP o un satélite. El rasgo semántico de la Actividad que causa el Resultado no es parte de la semántica léxica del verbo principal, sino un componente conceptual. Por ejemplo, la causa del cansancio en (3) se deriva a partir de implicaturas asociadas al conocimiento del mundo de 'dance' (“bailar”); en particular, su ejecución requiere un movimiento continuo e intencional del participante que conlleva un cansancio físico. En este sentido, KR representa la expresión de una relación semántico-conceptual en una estructura interclausal reducida, un tipo de estructura típicamente reservada a relaciones entre componentes semánticos altamente estructurados, es decir, parte de la estructura de la semántica verbal. Denominemos 'semántica mixta' a aquella que involucra, por un lado, componentes de la semántica estructural lexicalizada y, por otro, componentes del conocimiento del mundo activados mediante implicaturas convencionalizadas. Denominemos, además, ‘interfaz blanda' a aquella que permite la expresión de una 'semántica mixta’ en una estructura sintáctica típicamente reservada a argumentos y relaciones semánticas estructurales. El inglés exhibe con KR una interfaz blanda imposible en español.

Los adjetivos -y las propiedades que denotan - que pueden participar de KR no son numerosos, constituyen una lista muy restringida; esto indica que la productividad es limitada a la vez que le otorga a KR un matiz léxico. Finalmente, KR representa un quiebre de una interfaz sintaxis-semántica translúcida en la que el significado léxico del verbo principal rige en el dominio sintáctico local la cláusula: permite la introducción de objetos directos no habilitados por la semántica léxica del verbo principal.

\section{La dimensión tipológica}

¿Por qué existe KR en inglés? ¿Por qué no contamos con un equivalente en español? Con mayor precisión, nos preguntamos por qué solo existe en español (y de manera restringida) la versión más débil de KR como la ejemplificó (25) más arriba. Así enunciada, la pregunta tiene poco sentido ya que posiblemente toda lengua contiene una forma que no aparece en el inventario de otra lengua. Sin embargo, la pregunta adquiere sentido en el marco en el que la sitúo aquí: la idea es mostrar la consistencia de KR con la lógica interna del sistema gramatical inglés y, a su vez, su inconsistencia con el sistema español. París (2015) ofrece una motivación funcional basada en la tipología de Talmy (2000). En esta tipología el inglés es una ‘lengua de marco satelital' (sattellite-framed language) y el español, por el contrario, es una lengua de marco verbal (verb-framed language). En una [[situación]] observable de movimiento en el que un individuo camina y luego entra a un lugar, ese mismo estímulo es expresado en inglés por (29). 
(29) Bill walked into the pharmacy to buy some painkiller.

Bill caminó en-a la farmacia para comprar algún sedante

"Pedro entró a la farmacia a comprar algún sedante"

El inglés describe el [[evento $\left.{ }_{29}\right]$ ] mediante KR: un verbo de Actividad como matriz y una expresión preposicional introducen un Cambio Locativo como 'marco' — en el sentido de Talmytal que ambos eventos están en un mismo curso y en una relación de precedencia parcial. En contraste, ese marco es codificado en el verbo principal en la traducción al español en (29): "entrar" denota un Cambio Locativo puntual. La manera de movimiento no se codifica porque es predecible a partir del conocimiento del mundo y de reglas conversacionales (París, 2008). El núcleo del contraste es la elección del componente semántico expresado por el verbo matriz, en una lengua es Manera de movimiento mientras en la otra es un Cambio Locativo. París (2015) toma esta codificación como base para derivar la carencia de KR por su redundancia en el sistema lingüístico español. Si la Situación prototípica expresa el Resultado en el verbo matriz, carece de sentido contar con una Macrosituación KR específicamente dedicada a enriquecer la descripción en el verbo principal con un Resultado. Por el contrario, el verbo matriz en inglés expresa manera $\mathrm{y}$, por ende, KR se vuelve imprescindible para la lengua en la expresión de Resultado.

Levin y Rappaport (2019) aducen un factor léxico esencial para fundamentar la tipología de Talmy: Ia 'complementariedad disyuntiva Manera-Resultado'. Existe una clara tendencia universal en la codificación semántica tal que los verbos pueden describir o bien Manera o bien Resultado, pero no ambos al mismo tiempo. De hecho, existen ostensibles diferencias en los léxicos inglés y español (documentadas en, entre otros, Slobin (1996)). En particular, el inglés tiene muchos más verbos que el español en relación a la codificación de Maneras de movimiento; en contraste, muchos de los verbos de Cambio Locativo del inglés son latinos ("enter", "arrive”, “exit”). Esta complementariedad disyuntiva Manera-Resultado guarda un aspecto todavía más básico y central que la organización del léxico y la proyección sintaxis-semántica y es el de la complementariedad misma, es decir, la diferenciación Manera y Resultado y la aversión de los sistemas lingüísticos a hacerlos confluir en una misma unidad léxica. El problema es que las nociones de Manera y Resultado nunca han sido definidas más allá de la intuición. Esto ha permitido que, por ejemplo, Beavers y Koontz-Garboden (2012) y Goldberg (2010) señalen numerosos contraejemplos a la complementariedad: verbos de manera de matar ("guillotinar", "ahogar", "asfixiar", "fusilar", etc.) o manera de cocinar ("freír", "asar", "saltear", "dorar”, etc.). Es más, propongo que existe un conjunto extremadamente numeroso de verbos tradicionalmente interpretados como Realizaciones que violarían sistemáticamen-

6 La evidencia empírica no puede responder cuál es el origen de la tipología, si la interfaz sintáctico-semántica o la riqueza léxica. Desde un punto de vista analítico, sin embargo, la oración debería tener preeminencia por sobre el léxico. 
te la complementariedad definida en tales términos. En todos esos verbos la organización es siempre una secuencia Actividad-Cambio de Estado.

(30) Actividad $t_{1}<$ Cambio de Estado $t_{2}$

Se trata de verbos de movimiento, de creación y de consumición representados en (31), (32) y (33), respectivamente. Todos son Realizaciones dado que, por ejemplo, pueden ser modificados por una frase preposicional "en+tiempo".

(31) El avión planeó trece kilómetros en una hora.

(32) Mi compañero le escribió la carta al director en diez minutos.

(33) Pedro comió su almuerzo en cinco minutos.

Todos tienen una versión más simple, monoeventiva, que materializa una simple Actividad, como puede verse en (34), (35) y (36), respectivamente.

(34) El avión planeó sobre la ciudad durante una hora.

(35) Mi compañero escribió en su cama durante toda la siesta.

(36) La vaca comió pasto en la pradera durante todo el día.

La pregunta es ¿por qué no interpretamos que estas Realizaciones encarnan una estructura Manera-Resultado?? Por ejemplo, "escribir" en (32) especifica la Actividad del Actor y el Resultado, pero la literatura no interpreta que involucre una Manera y, sin embargo, no se ha explicitado una justificación. Mi propuesta es que el único desenlace posible aquí es la finalización (o no) de la carta, no existe una tercera alternativa y sin alternativas no se instancia Manera (Ernst, 2004).

Una Manera es una forma particular de hacer algo, es decir, es más específico que la pura noción de Actividad ${ }^{8}$. Es una Actividad que, o bien está subsumida en una Actividad más abs-

7 ¿Qué hace que una Actividad se torne una Realización? Las explicaciones varían sutilmente de teoría a teoría. Verkuyl (1993) asume que la semántica de algunos verbos viene con un rasgo específico y que solo cuando se combina con una frase nominal delimitada - [+QUA] — la descripción deviene una Realización. En Van Valin (2005) se trata de Realizaciones Activas, una derivación sistemática a partir de la pertenencia a una clase léxica. En todo caso, las teorías acuerdan que el punto de partida es una Actividad que deviene una Realización al asociarse un Cambio de Estado.

8 Se han propuesto definiciones de Manera en relación a los adverbios de Manera; típicamente todas captan la idea de una modificación interna del Evento que contempla alternativas y la relación parte-todo (Maienborn y Schäfer, 2011; Ernst, 2004: 58). Sin embargo, estas definiciones son demasiado generales como para captar la noción léxica de Manera en 'Verbo de Manera de'. 
tracta y general ("movimiento" subsume "caminar", "correr", etc.), o bien tiene un Resultado. Por ejemplo, "Ilorar" es simplemente Actividad, no es Manera, ya que no es una forma específica de hacer algo más general, ni tampoco tiene un Resultado asociado. En nuestra experiencia cotidiana no es infrecuente que se ejecute una Actividad que no esté conectada con una consecuencia específica. Pensemos en el Evento lexicalizado por "llorar". Primero, típicamente no tiene un argumento Afectado9. Por otro lado, puede haber un sinnúmero de consecuencias posibles luego del Ilanto de Pedro, no se puede determinar un único tipo de consecuencia que sea interpretable como Resultado. En este marco, la lexicalización de Actividad + Cambio de Estado en un único verbo sería sumamente ineficaz: deberíamos tener un verbo distinto para cada par "llorar + Cambio de Estado", por ejemplo, "llorar y sentir alivio" o "llorar y sentir vergüenza" o "llorar y lograr engañar a alguien”, etc. Si de captar patrones extralingüísticos se trata, es más eficaz desenganchar Actividad y Cambio de Estado y lexicalizarlos en distintos ítems léxicos. Esto es válido para el caso de "llorar”, pero también para un sinnúmero de Actividades.

El caso de los verbos de movimiento ejemplificados en (31) es especialmente interesante porque, a diferencia de (32) y (33), la literatura semántica los interpreta como verbos de Manera (de movimiento). Ahora bien, el tipo de Situación codificada por "caminar" no es intrínsecamente Manera, es simplemente un tipo de Actividad. La noción de Manera solo emerge cuando consideramos las alternativas de Movimiento. Manera siempre es 'Manera de', es una categoría relacional que aflora si la Actividad es una opción en un abanico de alternativas para ejecutar un concepto más general (p. ej., Movimiento) o bien obtener un Resultado. Para que haya alternativas tiene que satisfacerse como condición la separación entre Actividad y Resultado, no puede haber una sociedad fija uno-a-uno. Como se muestra en (33), no se requiere un Resultado especificado, es decir, el verbo "planear" es Manera de Movimiento aunque no se especifique un Resultado. En contraste, los verbos "escribir" en (31) o "comer" en (32) no son categorizables como Manera porque describen Actividades lo suficientemente generales como para no constituir una alternativa entre varias de ejecutar el mismo tipo de Actividad ([beber] es una Actividad bien distinta de [comer], mientras [dibujar] se distingue de [escribir]) y, además, ambas tienen un Resultado típico: "comer" entraña la consumición del Afectado mientras "escribir" entraña la creación de un objeto lingüístico completo"10. En

9 Aunque existe una versión quizás literaria de "Ilorar" que sí lo tiene:

(i) Pedro lloró a su madre durante años.

La Afectación del argumento "madre" es débil, sin embargo, y se limita a ser simplemente el Tema del Ilanto; no es un participante afectado por un Cambio de Estado.

10 "Devorar" puede ser analizado como un verbo de Manera mientras "comer" no lo es porque el primero entraña al segundo, es decir, si se devora algo, necesariamente se lo come tal que se especifican propiedades de la actividad de comer: se lo hace con mucha intensidad y en un periodo de tiempo más corto que el esperado. 
ambos casos, el Resultado aparece a través del completamiento de la Afectación del Paciente, pero si esta no se completa, no cambia la naturaleza del Evento.

Una pregunta central surge del análisis de (31) en términos de una Realización constituida por una Actividad clasificable como Manera de Movimiento. Si expresa una Actividad/Manera cerrada por un Cambio, lo que la convierte en una Realización, ¿por qué no considerarlo como una instancia de KR? Aske (1989: 6) fue el primero en advertir cierta distinción entre (27) y (28). El primero constituye una instancia de Telicidad en el sentido de imposición de un Límite a la Actividad que la convierte en un Evento; se trata de delimitar un Trayecto, es decir, insertar una Meta. En cambio, (27) especifica que ese Límite se alcanzó a través de traspasar un Contorno y, por lo tanto, el Tema se relocalizó en un Lugar; no es simplemente un Trayecto delimitado, sino un posicionamiento del Tema en un Lugar distinto, es un Cambio Locativo. En síntesis, KR exige más que Telicidad, requiere Cambio Locativo (lo cual entraña Telicidad).

En este punto podemos establecer ciertas conclusiones preliminares. Primero, los verbos que en cualquier lengua codifican Actividad y Evento Télico / Cambio de Estado / Locativo en un mismo curso de Situaciones no son una excepción. Segundo, no existe una diferencia intrínseca que distinga esas Actividades de las Actividades denominadas también Maneras, como tampoco la hay entre, por un lado, esos Eventos Télicos / Cambios de Estado / Locativo y, por otro, los Resultados. No hay una semántica verbal que sea intrínsecamente Manera o Resultado si no es en relación a un marco extrínseco constituible, primero, por una noción más general que la descrita por la Actividad y de la que esta es una alternativa y/o, segundo, por la posibilidad de obtener un mismo Resultado mediante tales alternativas. Los verbos de creación y consumición que codifican Realizaciones no violan la complementariedad porque no codifican Manera: el Resultado en estos verbos es la única salida o consecuencia posible que sigue a la Actividad específica descrita por el verbo (la única alternativa consiste en el grado de afectación del Tema incremental que puede ser total o parcial). Los verbos de Manera de Movimiento que devienen Télicos no codifican Resultado en sentido estricto (Cambio Locativo) y, por lo tanto, no puede considerarse que violen la Complementariedad. Por último, un verbo de Manera de Movimiento seguido por un Cambio Locativo constituye una instancia de KR (no es una codificación léxica, por lo que no viola la complementariedad).

De acuerdo a lo concluido hasta aquí, propongo aquí derivar la complementariedad Manera-Resultado propuesta por Rappaport y Levin (2010) de una condición cognitiva más básica: Ia ‘disociación’ Manera-Resultado. La idea es que separar Manera y Resultado es altamente eficaz en la tarea de constituir nuestra experiencia con el mundo extralingüístico. Las lenguas cuentan con esquemas semánticos universales que nos permiten captar Actividades - como "correr" - y Cambios - como "entrar" - independientemente el uno del otro. La confluencia de Manera y Resultado o, en términos más generales, Actividad y Cambio en un único ítem léxico A requiere que el [[evento]] en el mundo extralingüístico descrito por A satisfaga siempre - o, al menos, recurrentemente - la co-presencia de esa Actividad y ese Cambio. Si x ejecuta 
la actividad [[escribir una carta]], esa actividad co-ocurre con el cambio de estado total o parcial [[carta escrita]] a continuación. Si tiene lugar el Estado [[carta escrita]], necesariamente fue precedido de la actividad [[escribir la carta]]. Es económicamente eficaz tener un verbo que agrupe a ambos en su significado. Sin embargo, esta correlación está lejos de ser la más frecuente. Típicamente nos enfrentamos a acciones como [[reír]], [[nadar]] o [[disparar]] que no están seguidas necesariamente por un único tipo de Evento como Resultado. Sería enteramente antieconómico poblar el léxico con un verbo para cada asociación posible entre [[reír]] y los potenciales eventos que lo siguen. Igual razonamiento aplica a los Cambios, ya que pueden ser el Resultado de distintos tipos de Actividades.

\section{Teorías alternativas}

Dos propuestas sobre el contraste inglés-español en relación a KR que encarnan visiones alternativas a la defendida aquí son las minimalistas en Mateu (2012) y en Snyder (2012).

El eje de ambas teorías es que las lenguas como el español carecen de KR porque adolecen de un esquema sintáctico combinatorio que estaría presente en el inglés y que subyace a KR. En el caso de Mateu, ese esquema es conflation, un término adoptado de Talmy, pero reinterpretado sintácticamente y que es, precisamente, la estructura subyacente a KR. Consiste en la composición de una raíz con un verbo liviano nulo ("the compounding of a root with a null light verb" [Mateu, 2012: 255]). Ese verbo nulo ([Ø]) introduce el componente (semántico) change/go ('Cambio/Ir').

\section{FIGURA 2}

KR en el Minimismo

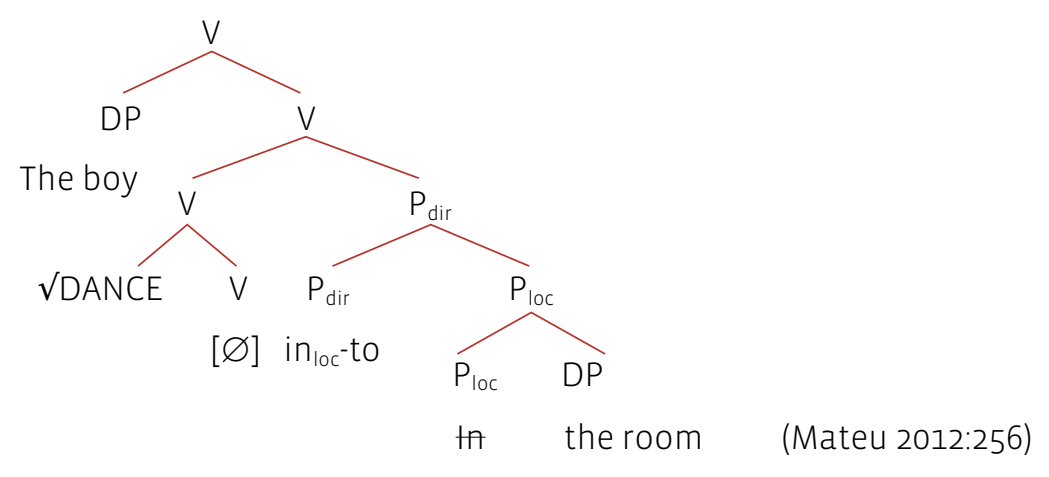

Esta es la representación subyacente a una oración KR como (37).

(37) The boy danced into the room.

El niño bailó en-a la habitación

"El niño entró a la habitación bailando" 
Obviando detalles, la clave es que al verbo nulo [ $\varnothing]$ se le permite 'confluir' — traducción tentativa de conflate - con la raíz "bailar" (VDANCE) y ese verbo nulo es interpretado como inacusativo, es decir, como un verbo intransitivo asociado a un Cambio. El español, entonces, no permitiría la confluencia de un verbo nulo inacusativo con un verbo de Manera.

La introducción de un verbo liviano nulo a KR es un intento, acertado o no, de captar la irrupción de la semántica de Cambio, ausente en el verbo principal. Criticar por artificial la propuesta de un verbo nulo constituiría una crítica externa, es decir, un rechazo al modelo y práctica del minimalismo y, por lo tanto, resulta una crítica irrelevante. La crítica que ensayo aquí es, por el contrario, interna. En primer lugar, el análisis de Mateu no registra el fenómeno formal más saliente de KR y que consiste en la presencia de un objeto directo no habilitado por el verbo que funciona como núcleo de la frase verbal y que, por lo tanto, domina sintácticamente a esa FN. Los verbos nulos no pueden tener estructura argumental y, por ende, es impensable que habilite tal objeto directo. Por otro lado, el español sí tiene fusión de predicados o nuclear juncture o, simplemente, V-V (fenómeno originalmente denominado "unión de cláusulas" por Aissen y Pelmutter [1983]). Cierto subtipo de la construcción causativa con "hacer" ha sido analizada como nuclear juncture (Ackerman y Moore, 1999; París, 2012), así como también los verbos de 're-análisis' como "querer" en contextos de subida de clíticos ("Pedro lo quiere limpiar"). Sin embargo, a diferencia de KR, nuclear juncture en español solo combina un verbo liviano con otro pleno o dos verbos tal que uno habilita semánticamente al otro (su complemento). Por el contrario, KR combina dos predicados plenos tal que ninguno habilita semánticamente al otro. Estas propiedades esenciales del contraste no son captadas en el trabajo de Mateu. Incluso se puede cuestionar que el predicado nulo que introduce el Cambio se fusiona con el verbo principal produciendo una expresión como "bailar+Cambio" en relación a (3). Sin embargo, dado que *John danced Liz (“John bailó a Liz") es agramatical, parece apropiado asociar el Cambio a la FA tired. Por último, la propuesta de Mateu no deriva la presencia o carencia de la estructura que propone para describir a KR de, respectivamente, las organizaciones internas de los sistemas inglés y español. Este es, sin embargo, el objetivo de Snyder (2012), quien propone un criterio formal para explicar la presencia de KR en algunas lenguas y su ausencia en otras. Esta propuesta está sujeta a todas las críticas hechas al trabajo ya discutido, pero, aun siendo menos precisa que la de Mateu, tiene la virtud de enmarcar el contraste en propiedades generales de los sistemas tal que la presencia o carencia de KR se derivaría de propiedades arquitectónicas generales de las lenguas. El criterio es el 'parámetro de la composición' con el que se intenta explicar la presencia a la vez de compuestos nominales productivos y KR en inglés y la ausencia de ambos en español. En su versión más formal (Snyder, 2012: 285) define al parámetro de composición en los siguientes términos:

a. Un lenguaje permite o no permite Modificación Generalizada.

b. Modificación Generalizada: Si $\alpha$ y $\beta$ son nodos sintácticos hermanos bajo el nodo $\gamma$, y $\alpha$ es el núcleo de $\gamma$, si $\alpha$ denota un clase de individuos, $\gamma$ denota un subtipo o miembro de esa clase individuos que, además, sostiene una relación pragmática apropiada con la denotación de $\beta$. 
Snyder predice que el español no cuenta con un equivalente a KR del inglés porque no instancia este parámetro. Sin embargo, definido así, ese parámetro es capaz de describir la estructura de la construcción adverbial de gerundio en español ejemplificada en (38), es decir, el "llegar" describe un tipo de Evento y "llegar corriendo" es una subclase de "llegar" al tiempo que es muy apropiado interpretar a "correr" como una manera de "llegar".

(38) El niño llegó a la escuela corriendo.

Si bien el parámetro de la composición es inviable como explicación, Snyder apunta a un fenómeno general que, aun cuando no sea captado por su principio, resulta intuitivamente plausible. Interpreto ese fenómeno en términos muy abstractos como diferentes tendencias en las estrategias combinatorias del inglés y el español. Por ejemplo, los compuestos nominales del inglés como en (39) cubren un espectro de posibles interpretaciones.

(39) Frogman = Un hombre (con apariencia de sapo) v (que camina como un sapo) v (que tiene un sapo al lado) v (que vende sapos) v (el más rápido en un grupo para cazar sapos) v ....

Este constructo gramatical pone dos nombres juntos que denotan conjuntos de cosas diferentes y la gramática no restringe la relación al punto que ciertos compuestos son exocéntricos (pickpocket). Este espectro combinatorio abierto es poco tolerable para el español, que tiende a indicar la relación semántica con algún componente explícito del sistema, aun cuando sea también semánticamente vago, como la preposición "de”, o a asignar un único significado. Un "hombre-sapo" no existe en español, y de ser acuñado, se interpretaría solo como un sapo con mente humana o una mente humana en el cuerpo de un sapo. Alternativamente, "hombre-rana" sí existe, pero tiene un estatuto léxico con un solo significado posible.

El análisis de este ejemplo me lleva a sugerir que el rol de la forma -rasgos formales o gramaticales - en la combinatoria tiene notoriamente más incidencia en español que en inglés. Esto coincide con el hecho indiscutible de la mayor riqueza y complejidad morfológica del español frente al inglés. En inglés la palabra viene del léxico casi ‘desnuda’ a combinarse sintácticamente; en español, por el contrario, la palabra llega a la combinatoria sintáctica abundantemente 'arropada' de morfología. No son procesos combinatorios idénticos. En inglés el trabajo que tiene que realizar el hablante/oyente para determinar la relación semántica entre las dos palabras se ejecuta enteramente 'on line'. Por el contrario, el español condiciona estrechamente esa relación con rasgos léxico-formales. El diseño de los dos signos es diferente. En tal sentido, el rol de la recuperación léxica - y, por lo tanto, de la memoria a largo plazo - es más fuerte en español, mientras que en inglés el trabajo duro tiene lugar durante la combinatoria 'on line', por lo que involucra la memoria de trabajo. Podemos captar esa diferencia diciendo que la combinatoria entre signos de una misma categoría (clase de palabras) en español es cerrada: Ios rasgos formales tienden a constreñir su articulación a una sola semántica. En inglés la combinatoria entre palabras de la misma categoría léxica 
tiende relacionarse con una semántica abierta: las potenciales relaciones semánticas entre dos palabras al interior de una frase suelen incluir un conjunto abierto de posibilidades ${ }^{11}$. En términos de KR, la semántica abierta es lo que caracterizamos más arriba como la realización de una 'semántica mixta’ — mezcla de estructura semántica con información conceptual—en una sintaxis reducida mediante una 'interfaz blanda', interfaz imposible en español.

\section{Conclusión}

El contraste inglés-español registra la presencia del subtipo más débil y menos representativo de KR en las dos lenguas mientras el amplio espectro de subtipos de KR solo está presente en el inglés. La versión compartida es aquella en la que el Resultado no es introducido por un adjunto sino que este es introducido por la semántica del verbo principal y aquel solo se limita a especificarlo. Por el contrario, KR en inglés es fecunda en variantes y recurrente en frecuencia a la vez que constreñida por patrones de interfaz sintaxis-semántica. La presencia de KR y su carencia coocurren con diferentes rasgos fundamentales de los sistemas inglés y español. En primer lugar, KR cohabita en el sistema inglés con una Situación prototípica que, dada a describir una [[situación]] que contiene una [[actividad]] seguida por un [[cambio]], proyecta una [Actividad] como verbo principal mientras el [Evento] es registrado por un elemento formalmente reducido y dependiente. Por el contrario, la carencia de KR se correlaciona con una Situación española en la que el Evento es introducido por el verbo principal. Se propuso un análisis en el que tales coocurrencias son consistentes ya que reportan divisiones del trabajo dentro de cada sistema tal que lo que no es ejecutado por la Situación prototípica de una lengua lo lleva a cabo una Macrosituación complementaria: KR en inglés, la construcción adverbial de gerundio en español. Estas complementariedades tienen su raíz en una tendencia del léxico a registrar Actividades y Eventos en distintas unidades léxicas. Esto es así incluso cuando el [[evento]] sigue a la [[actividad]] en el mundo extralingüístico a condición de que no lo haga siempre, es decir, que la [[actividad]] suela aparecer también seguida por [[eventos]] de distinto tipo. Es un hecho de nuestra experiencia del mundo extralingüístico que no siempre de una [[actividad]] se sigue un único tipo de resultado y sería ineficaz por antieconómico que las lenguas registraran con una unidad léxica diferente cada asociación posible de una [[actividad]] con distintos resultados. Al principio cognitivo que capta esta característica del mundo extralingüístico en relación a nuestra manera de concebirlo lo he denominado ‘disociación’ y lo presento como la base cognitiva de la tendencia a separar en el léxico [Actividad] y [Evento] o, más específicamente, [Manera] y [Resultado]. Otra propiedad de la

11 Es tentador arriesgar una asociación entre abierto y cerrado con, respectivamente, el mayor costo en la recuperación léxica en la memoria de largo plazo y, por el contrario, con el mayor costo a nivel de la combinatoria producida en la memoria de trabajo. En términos neurolingüísticos, se podría decir que el español es una lengua N400 (el potencial generado en la recuperación léxica) mientras el inglés es una lengua P-600 (el potencial generado a partir de la combinación de palabras en frases y oraciones). 
semántica de los subtipos fuertes de KR es que instancia una noción de causalidad con rasgos que la diferencian del primitivo léxico CAUSA, ese inherente a verbos como "matar" o "romper". En los cursos causales de Situaciones propios de KR, una Actividad altamente especificada (es decir, descrita por el verbo principal) afecta gradualmente a un participante hasta causar su Cambio de Estado especificado por, por ejemplo, un adjetivo en una AP. La categoría CAUSA, en cambio, remite a contenidos que no especifican la actividad del participante Agente, solo acarrean el entrañamiento de que es el responsable de un Cambio de Estado que sí es especificado. La noción de 'curso causal de Situación' es más específica en cuanto entraña el solapamiento temporal de una Actividad y un Cambio de Estado gradual (escalar). En síntesis, ciertos casos de KR pueden involucrar la relación semántica CAUSA, pero otros, los más típicos, involucran otro tipo de relación causal.

Las propiedades formales de KR se condicen con el estilo combinatorio del inglés y, por el contrario, resultan extrañas al sistema español. En particular, KR muestra una potestad débil del verbo principal de la oración respecto de su dominio local al punto de permitir la introducción de objetos directos no habilitados por su semántica léxica pero que, a la vez, son insertados en esa semántica. Esta situación es improbable en español con dos predicados plenos. El español no admite sin más la combinación de dos elementos léxicos plenos en un marco semánticamente abierto; por el contrario, requiere que la gramática condicione a la relación entre ambos, tal que la semántica que se combina es la estructuralmente lexicalizada, no aquella asociada conceptual y/o pragmáticamente. Por el contrario, el inglés contiene estructuras como los compuestos nominales en los que la relación semántica entre ambos es extremadamente abierta al punto de ser mixta, es decir, combinar semántica estructural y conceptual. Por otro lado, sabemos que las nociones de objeto indirecto y objeto directo — parte esencial del dominio local del verbo principal- son mucho más débiles en inglés que en español. El supuesto objeto indirecto puede ser sujeto de una pasiva en inglés pero no en español y el orden entre ambos objetos es intercambiable en inglés con efectos semánticos pero no en español. El inglés consta, entonces, de 'primary object' y 'secondary object', lo que hace que un participante típicamente expresado como objeto indirecto en español pueda ser 'primary object' (John sent Liz flowers "John le envió flores a Liz").

Estos comportamientos formales pueden ser caracterizados diciendo que dos elementos de la misma categoría léxica se combinan en inglés mediante una interfaz blanda que permite un conjunto de relaciones semánticas. En cambio, en español esa combinatoria se da en el marco de una interfaz cerrada a idealmente una sola relación semántica.

\section{Bibliografía citada}

Ackerman, Farrel, y John Moore, 1999: "Syntagmatic and paradigmatic dimensions of cause encodings”, Linguistics and Philosophy 22 (1), 1-44.

Aissen, Judith, y David Perlmutter, 1983: "Clause Reduction in Spanish" en David Perlmutter (ed.): Studies in Relational Grammar, Chicago: University of Chicago Press, 360-403. 
Aske, John, 1989: "Path Predicates in English and Spanish: a Closer Look", Berkeley Linguistic Society $15,1-14$.

Beavers, John, y Andrew Koontz-Garboden, 2012: "Manner and Result in the Roots of Verbal Meaning", Linguistic Inquiry 43 (3), 331-369.

BoAs, Hans, 2000: The Resultative Construction in English and German. Tesis doctoral, University of North Carolina, Chappel Hill.

ERnst, Thomas, 2004: The Syntax of Adjuncts, Cambridge: CUP.

Goldberg, Adele, 2010: "Verbs, Constructions, and Semantic Frames" en Malka Rappaport Hovav, Edith Doron e Ivy Sichel (eds.): Lexical Semantics, Syntax, and Event Structure, Oxford: Oxford University Press, 39-59.

Goldberg, Adele, y Ray JaCKEndoff, 2004: "The English Resultative as a Family of Constructions", Language 80 (3), 532-568.

KRIFKA, Manfred, 1998: "The Origins of Telicity" en Susan RothsteIn (ed.): Events and Grammar, London: Kluwer, 197-235.

Levin, Beth, y Malka Rappaport-Hovav, 2019: "Lexicalization Patterns" en Robert Truswell (ed.): Handbook of Event Structure, Oxford: Oxford University Press, 395-425.

Levin, Beth, y Malka Rappaport-Hovav, 1995, Unaccusativity: At the Syntax-Lexical Semantics Interface, Cambridge, MA: MIT Press.

LEwIS, David, 1973: Counterfactuals, Cambridge, MA: Harvard University Press.

Maienborn, Claudia, y Martin Schäfer, 2011: "Adverbs and Adverbials" en Claudia Maienborn, Klaus Heusinger y Paul Portner (eds.): Semantics. An International Handbook of Natural Language Meaning. Volume 1, Berlin: Mouton de Gruyter, 1390-1420.

MATEu, Jaume, 2012: "Conflation and incorporation processes in resultative constructions" en Violeta Demonte y Louise McNally (eds.): Telicity, Change, and State, Oxford: Oxford University Press, 252-278.

PARis, Luis, 2006a: "Implicating and Focusing on Underspecified Lexical Information" en Klaus von Heusinger y Ken Turner (eds.): When Semantics Meets Pragmatics, Oxford: Elsevier, 421-444.

PARIS, Luis, 2006b: “La codificación gramatical de eventos: Medio”", Signos 39, 259-262. 
Paris, Luis, 2008: "On Expanditures” en Nikki Adams, Alice Cooper, Fey Parrill y Thomas Wier (eds.): Proceedings of the $40^{\text {th }}$ Regional Meeting of the Chicago Linguistic Society Conference, Chicago: CLS, 291-304.

PARIS, Luis, 2012: "Sintaxis y Semántica de la construcción causativa" en Pedro MaIRAL, Lilian GueRRERO y Carlos VerGara (eds.): Avances en la Gramática del Rol y la Referencia, Madrid: Editorial Akal, 287-307.

Paris, Luis, 2015: “Preferencia aspectual en el léxico y compensación morfo-sintáctica: el contraste inglés-español”, Revista de Lingüística Teórica y Aplicada 53 (1), 59-81.

PARis, Luis, 2016: "Actor y Paciente en inglés y español”, ponencia presentada en el Primer CoIoquio de Investigaciones en Gramática y Léxico. Mendoza, abril.

PARIS, Luis, 2018: "The Syntax of the Directed Motion Subtype of the Spanish Gerund Construction" en Rolf Kalluweit, Lisann Künkel and Eva Staudinger (eds.): Applying and Expanding Role and Reference Grammar, Freiburg: NIHI [disponible en http://doi.org/10.6094/978-3928969-60-4].

RAPPAPORT, Malka, y Beth LeVIN, 2010: "Reflections on Manner/Result Complementarity" en Malka RaPPAPORT, Edith Doron e Ivy Sichel (eds.): Lexical Semantics, Syntax, and Event Structure, Oxford: Oxford University Press, 21-39.

RaPPAPORT, Malka, y Beth Levin, 2001: "An event structure account of English resultatives", Language 77 (4), 766-779.

SLobin, Dan, 1996: "Two ways to travel: Verbs of motion in English and Spanish" en Masayoshi Shibatani y Sandra Thompson (eds.): Grammatical constructions: Their form and meaning, Oxford: Clarendon Press, 195-220.

Snyder, William, 2012: "Parameter theory and motion predicates" en Violeta Demonte y Louise McNally (eds.): Telicity, Change, and State, Oxford: Oxford University Press, 279-299.

TALmy, Leonard, 2000: Toward a Cognitive Semantics, Cambridge, MA: MIT Press.

VAN ValIN, Robert, 2005: Exploring the Syntax-Semantics Interface, Cambridge: CUP.

VERKUYL, Henk, 1993: A theory of aspectuality. The interaction between temporal and atemporal structure, Cambridge: Cambridge University Press.

Washio, Ryuichi, 1997: "Resultatives, compositionality, and language variation”, Journal of East Asian Linguistics 6, 1-49. 